\title{
Effect of Surgical Caseload on Revision Rate Following Total and Unicompartmental Knee Replacement
}

\author{
Alexander D. Liddle, DPhil, MRCS, Hemant Pandit, DPhil, FRCS(Orth), Andrew Judge, PhD, and \\ David W. Murray, MD, FRCS \\ Investigation performed at the Nuffield Department of Orthopaedics, Rheumatology, and Musculoskeletal Sciences, \\ University of Oxford, Oxford, England
}

\begin{abstract}
Background: High-volume surgeons attain the best results following unicompartmental knee replacement (UKR), but the exact relationship between caseload and outcome is not clear. It is not known whether this effect is due to patient selection or surgical skill nor whether a similar effect is seen in total knee replacement (TKR). The aim of this study was to quantify the effect of surgical caseload on survival of both TKR and UKR.
\end{abstract}

Methods: This study was based on 459,280 patient records (422,149 TKRs and 37,131 UKRs) from the National Joint Registry for England and Wales. The caseload-outcome relationship was characterized graphically and quantified using regression techniques. Patient selection was compared among high, medium, and low-volume surgeons. Prosthetic survival was compared between UKRs (performed by high, medium, and low-volume surgeons) and matched TKRs.

Results: Caseload affected survival of TKR and, more strongly, of UKR. The revision rate following UKR dropped steeply until the volume reached ten cases per year, plateauing at thirty cases. For surgeons performing fewer than ten UKRs per year, the mean eight-year rate of survival of the UKRs was $87.9 \%(95 \%$ confidence interval $[\mathrm{Cl}]=86.9 \%$ to $88.8 \%)$ compared with $92.4 \%(95 \% \mathrm{Cl}=90.9 \%$ to $93.6 \%)$ for those who performed thirty UKRs or more per year. Analysis of the TKRs showed a linear decrease in revision rate as caseload increased (hazard ratio [HR] for revision $=0.99[95 \% \mathrm{Cl}=0.98$ to 0.99 ] for every five-case increase in caseload). Surgeons who performed a lower volume of UKRs tended to operate on younger and healthier patients and were more likely to perform revisions to treat loosening and pain. After matching of patients who had undergone UKR with those who had undergone TKR, the surgeons who performed a high volume of UKRs were found to have an eight-year revision/revision rate similar to that seen after TKR (HR for revision or reoperation $=1.10$ [95\% $\mathrm{Cl}=0.99$ to 1.22 ] favoring TKR).

Conclusions: This study confirmed the importance of surgical caseload in determining the survival of UKR and, to a lesser extent, TKR. The reasons for this effect are complex and not fully explained by variables recorded in the National Joint Registry; however, the patient selection and revision threshold of lower-volume surgeons may be a factor. Examination of matched patients in this study demonstrated that high-volume surgeons can achieve revision/reoperation rates similar to those observed following TKR.

Level of Evidence: Therapeutic Level III. See Instructions for Authors for a complete description of levels of evidence.

Peer Review: This article was reviewed by the Editor-in-Chief and one Deputy Editor, and it underwent blinded review by two or more outside experts. It was also reviewed by an expert in methodology and statistics. The Deputy Editor reviewed each revision of the article, and it underwent a final review by the Editor-in-Chief prior to publication. Final corrections and clarifications occurred during one or more exchanges between the author(s) and copyeditors.

Disclosure: One or more of the authors received payments or services, either directly or indirectly (i.e., via his or her institution), from a third party in support of an aspect of this work. In addition, one or more of the authors, or his or her institution, has had a financial relationship, in the thirty-six months prior to submission of this work, with an entity in the biomedical arena that could be perceived to influence or have the potential to influence what is written in this work. No author has had any other relationships, or has engaged in any other activities, that could be perceived to influence or have the potential to influence what is written in this work. The complete Disclosures of Potential Conflicts of Interest submitted by authors are always provided with the online version of the article. 
The Journal of Bone \& Joint Surgery - Jbjs.org Volume 98-A • Number 1 · January 6, 2016
Effect of Surgical Caseload on Revision Rate After Total and Unicompartmental Knee Replacement
$\mathrm{T}$ he use of unicompartmental knee replacement (UKR) remains controversial ${ }^{1}$. While it has well-described advantages, including a lower rate of morbidity and mortality ${ }^{2-4}$, faster recoveries, and superior patient-reported outcomes ${ }^{5-7}$, national joint registries have consistently demonstrated UKR to have a higher revision rate than total knee replacement (TKR) ${ }^{6,8-11}$.

The revision rate after UKR varies among surgeons and units. Long-term studies by high-volume surgeons, from centers involved in designing the prostheses and elsewhere, have reported prosthetic survival rates comparable with those of $\mathrm{TKR}^{12-16}$, whereas lower-volume surgeons and national joint registries have reported revision rates more than double that of $\mathrm{TKR}^{17-19}$. This suggests that the risk factors for revision following UKR are modifiable: if other users of UKR adopt the same practices as the high-volume users, the advantages of UKR could be gained without paying the price of an unacceptably high revision rate.

Much of the focus on surgical practice related to UKR has concerned surgical caseload: the number of UKRs performed per surgeon (or per unit) per year. Studies of three national joint registries have demonstrated a relationship between the revision rate following UKR and surgical (or unit) case$\operatorname{load}^{6,20,21}$. However, caseload itself does not predict outcome; rather, it is the expression of other predictors. The most obvious of these is surgical skill; however, low-volume surgeons have reported excellent results with $\mathrm{UKR}^{22}$, and some surgeons who perform a high volume of TKRs have failed to attain acceptable results with $\mathrm{UKR}^{23}$. Other factors may include revision

TABLE I Patient Characteristics in the TKR Group and the UKR Volume Groups

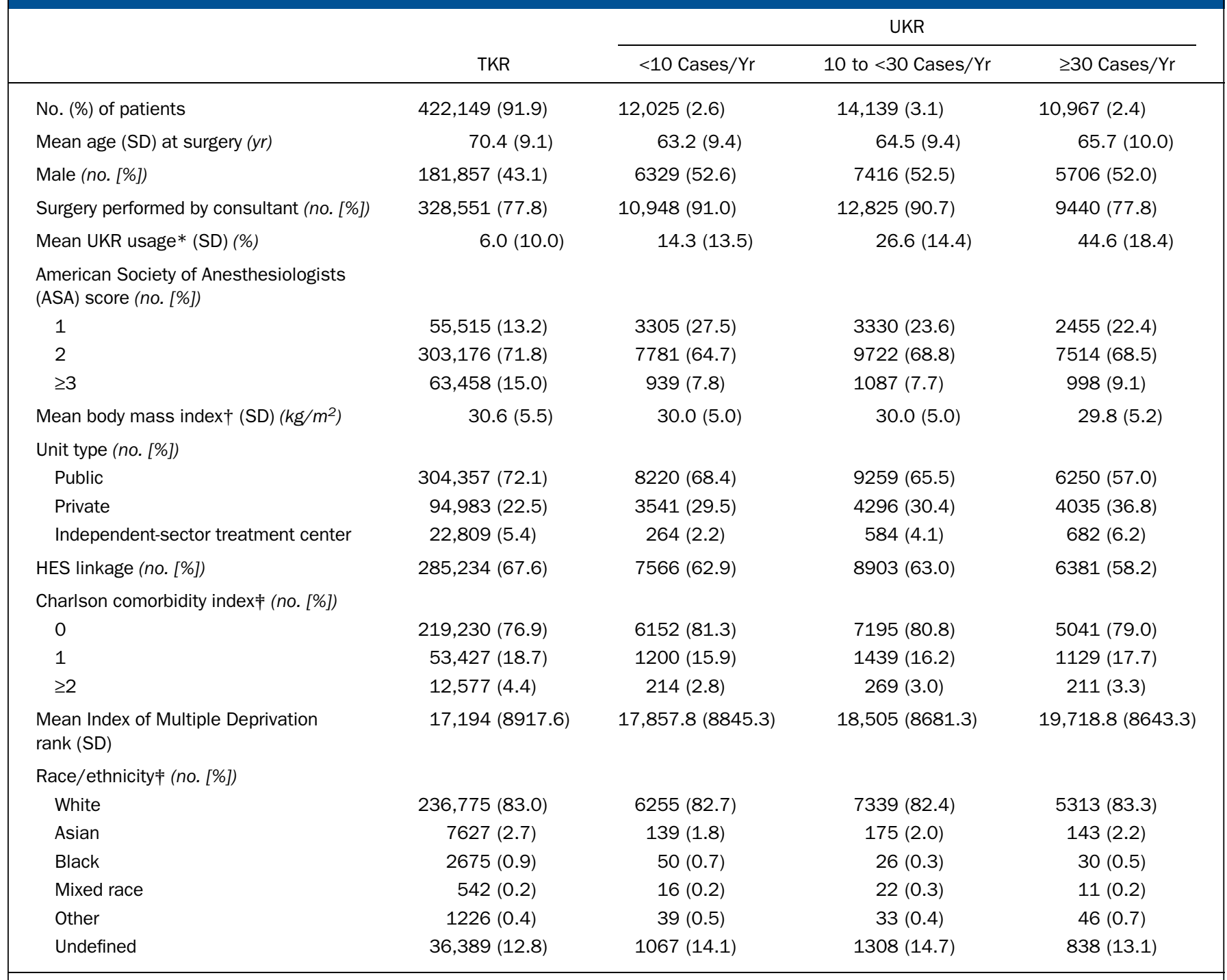

*The proportion of the surgeon's knee replacements that were UKRs. †Missing for $52.7 \%$ of the patients. $¥$ Values were derived from the HES dataset and so represent proportions of the HES-linked cohort. 
The Journal of Bone \& Joint Surgery - Jbjs.org Volume 98-A • Number 1 · January 6, 2016
Effect of Surgical Caseload on Revision Rate After

Total and Unicompartmental Knee Replacement

\begin{tabular}{|c|c|c|}
\hline \multirow[b]{2}{*}{ Spline Section } & \multicolumn{2}{|c|}{$\begin{array}{l}\text { HR per } 5 \text {-Case Increase in Caseload } \\
\quad \text { Within Spline Section }(95 \% \mathrm{Cl})\end{array}$} \\
\hline & TKR & UKR \\
\hline Overall & $0.99(0.98-0.99)$ & \\
\hline $0-10$ cases & & $0.86(0.78-0.94)$ \\
\hline $11-29$ cases & & $0.90(0.86-0.93)$ \\
\hline$\geq 30$ cases & & $1.00(0.98-1.02)$ \\
\hline
\end{tabular}

threshold (surgeons who rarely use UKR may be more ready to perform a revision than high-volume users of UKR $)^{24}$ and patient selection. Caseload has been found to have a similar effect on the results of TKR, but the magnitude of that effect has not been compared with the effect in UKR ${ }^{25-27}$.

The aim of this study was to answer these questions. The analysis was performed in three parts. First, a descriptive analysis was conducted to delineate the effect of caseload on the survival of UKR and to assess the degree to which this effect is also present in TKR. These data were used to determine evidence-based thresholds to indicate high, medium and low-UKR-caseload groups. In the second analysis, the characteristics of the three caseload groups were compared to determine the extent to which differences in patient selection explain the caseload effect in UKR. In the third analysis, the outcomes of UKR performed by high, medium, and lowvolume surgeons were compared with those of matched patients who had undergone TKR.

\section{Materials and Methods \\ Data Sources}

Tn this study, we used data from two sources, the National Joint Registry for 1 England and Wales and England's Hospital Episode Statistics (HES) database. The National Joint Registry was used as the master database, as it contains the most comprehensive details of the surgical procedures and linked revision data. Patients were matched between databases by using at least three of the following unique identifiers: National Health Service (NHS) identity number, date of birth, sex, and postal code.

The effect of caseload on implant survival was examined using an extract of 552,015 patient records from the National Joint Registry. This sample comprised all primary knee replacements recorded from the National Joint Registry's inception in 2003 to the extraction date (August 2012). Candidates for inclusion were adults who had undergone primary UKR or TKR for osteoarthritis. Patients who underwent patellofemoral replacement or "complex primary" operations, and those with missing operative details, were excluded. As the first and last years of data collection were incomplete, cases from 2003 and 2012 were excluded. The study group comprised 459,280 cases, of which 37,131 (8.1\%) were UKRs.

The HES database was used for the analysis of patient factors (in the second part of the study) and the matching of patients for the third part. HES contains information on every inpatient stay in NHS hospitals in England. Patients undergoing surgery in private units or in Wales are not included. As this reduces the number of cases, HES was not used in the descriptive analysis; however, a large amount of additional data is available for patient records linked to HES, allowing very close matching. HES includes detailed socioeconomic and comorbidity data, perioperative complications, and non-revision reoperations.

\section{Exposures and Outcomes}

Each surgeon in the National Joint Registry has a specific identifier, used in both private and public hospitals. With use of this identifier, each surgeon's UKR caseload was calculated for each calendar year. The mean caseload (cases per year) for each surgeon was then calculated, excluding the years in which the surgeon performed no UKRs (preventing an artificial reduction in the caseloads of surgeons who started contributing after the start of data collection or who retired or stopped performing UKRs in later years). Each patient was allocated a value representing the caseload of the surgeon who was in charge of his/her operation in the year in which the surgery was performed.

TABLE III Reasons for Revision of UKRs in Low, Medium, and High-Volume Groups

\begin{tabular}{|c|c|c|c|c|c|c|c|c|c|}
\hline & \multicolumn{3}{|c|}{$<10$ Cases/Yr } & \multicolumn{3}{|c|}{10 to $<30$ Cases $/ Y r$} & \multicolumn{3}{|c|}{$\geq 30$ Cases/Yr } \\
\hline Aseptic loosening & 281 & 32.4 & 2.3 & 181 & 24.5 & 1.3 & 111 & 29.8 & 1.0 \\
\hline Unexplained pain & 195 & 22.5 & 1.6 & 168 & 22.7 & 1.2 & 53 & 14.2 & 0.5 \\
\hline Disease progression & 89 & 10.3 & 0.7 & 95 & 12.9 & 0.7 & 50 & 13.4 & 0.5 \\
\hline Malalignment & 60 & 6.9 & 0.5 & 34 & 4.6 & 0.2 & 17 & 4.6 & 0.2 \\
\hline Dislocation & 50 & 5.8 & 0.4 & 60 & 8.1 & 0.4 & 41 & 11.0 & 0.4 \\
\hline Infection & 39 & 4.5 & 0.3 & 38 & 5.1 & 0.3 & 21 & 5.6 & 0.2 \\
\hline Instability & 36 & 4.1 & 0.3 & 35 & 4.7 & 0.2 & 14 & 3.8 & 0.1 \\
\hline Wear & 14 & 1.6 & 0.1 & 16 & 2.2 & 0.1 & 7 & 1.9 & 0.1 \\
\hline Implant fracture & 0 & 0.0 & 0.0 & 3 & 0.4 & 0.0 & 0 & 0.0 & 0.0 \\
\hline
\end{tabular}


TABLE IV Matched Survival Comparisons

\begin{tabular}{|c|c|c|c|c|c|c|}
\hline & \multicolumn{2}{|c|}{$<10$ Cases/Yr } & \multicolumn{2}{|c|}{10 to $<30$ Cases $/ \mathrm{Yr}$} & \multicolumn{2}{|c|}{$\geq 30$ Cases/Yr } \\
\hline Matching pool with HES linkage (no.) & 285,234 & 8367 & 285,234 & 8903 & 285,234 & 6316 \\
\hline No. matched & 24,603 & 8201 & 26,388 & 8796 & 18,672 & 6224 \\
\hline$\%$ matched & 8.6 & 98.0 & 9.3 & 98.8 & 6.5 & 98.5 \\
\hline $\begin{array}{l}\text { Survival rate }(95 \% \mathrm{Cl}) \text { with revision* } \\
\text { only as end point (\%) }\end{array}$ & $95.8(95.3-96.3)$ & $87.2(85.8-88.4)$ & $96.2(95.7-96.6)$ & $89.8(88.3-91.0)$ & $96.2(95.4-96.8)$ & $91.2(88.8-93.1)$ \\
\hline $\begin{array}{l}\text { Survival rate }(95 \% \mathrm{Cl}) \text { with revision/ } \\
\text { reoperationt as end point (\%) }\end{array}$ & $86.4(85.6-87.2)$ & $77.5(75.8-79.1)$ & $87.2(86.4-88.0)$ & $81.2(79.4-82.8)$ & $88.3(87.1-89.3)$ & $83.1(79.9-85.8)$ \\
\hline HR $(95 \%$ Cl) for revision/reoperation & \multicolumn{2}{|c|}{$1.61(1.50-1.73), p<0.001$} & \multicolumn{2}{|c|}{$1.32(1.22-1.43), p<0.001$} & \multicolumn{2}{|c|}{$1.10(0.99-1.22), p=0.086$} \\
\hline
\end{tabular}

UKR or TKR survival was calculated per surgeon per year and expressed as revisions per 100 implant-years ${ }^{9}$. A Kaplan-Meier estimate was produced to compare implant survival among the case-volume groups. Survival analysis was censored at eight years; this reflects the small numbers included in the National Joint Registry during its early years. All values presented in this report relate to eight-year survival.

\section{Analysis}

For the descriptive analysis, the effect of caseload on the revision rate was characterized using scatterplots, and curves were fitted using locally-weighted scatterplot smoothing (LOWESS) ${ }^{28}$. For clarity, the scatter points were suppressed in the plots presented. As these plots demonstrated a nonlinear effect of UKR caseload on implant survival, three linear splines were fitted, corresponding to roughly linear segments observed on the plots. Once the splines were fitted, survival rates in each segment were compared using Cox regression.

In the second analysis, cases were allocated to three groups corresponding to each spline section, representing low, medium, and high UKR usage. Patient characteristics in each group were compared using linear and logistic regression, and multivariable regression was undertaken to determine whether the differences in patient factors among the groups explained the difference in outcomes. In the final analysis, the UKRs in each of the volume groups were compared with TKRs in matched patients. Both revisions and non-revision reoperations (such as manipulation under anesthesia for stiffness, arthroscopy, amputation, and arthrodesis) could be assessed using HES data, and both revision and revision/reoperation (which may be a better indicator of failure than revision alone) were used as end points in the survival analysis. Matching was performed using propensity score methods in order to adjust for baseline differences in patient characteristics and to address the issue of confounding by indication. Full details of the propensity score matching are given in the Appendix. All statistical analyses were performed using Stata/IC (version 12 for Windows; StataCorp).

\section{Source of Funding}

This study was funded by Arthritis Research UK (grant number 20499) and the Royal College of Surgeons of England. Neither body was involved in the conduct of the study.

\section{Results}

Corty-nine percent (1272) of the 2589 surgeons listed in the F National Joint Registry as performing a knee replacement had performed at least one UKR between 2004 and 2012 (Fig. 1). Users of UKR performed an average of 5.4 per year (standard deviation $[\mathrm{SD}]=9.3$ ), with $25 \%$ (313 of 1272) having a mean caseload of only one UKR per year in the years that they performed UKR; this was the most common mean UKR caseload. As higher-volume surgeons perform more operations by definition, the mean number of cases per year on the patient level was higher $(24.8[\mathrm{SD}=25.0])$. Increases in caseload were accompanied by an increase in the proportion of the surgeon's knee replacements that were UKRs (termed "usage" of UKR).

Ninety-eight percent (2536) of the 2589 surgeons had performed at least one TKR; the mean number of TKRs per year was $33.6(\mathrm{SD}=32.5)$ on the surgeon level and $70.5(\mathrm{SD}=49.6)$ on the patient level. Baseline patient characteristics are given in Table I.

\section{Descriptive Analysis}

Overall, the unadjusted eight-year implant survival rate was $97.1 \%$ (95\% confidence interval $[\mathrm{CI}]=97.0 \%$ to $97.2 \%)$ for TKR and $89.8 \%$ (95\% CI $=89.1 \%$ to $90.4 \%)$ for UKR. For both operations, increasing caseload was associated with improving implant survival. However, this effect was much greater for UKR (Fig. 2). With UKR, there was an initial steep drop in the

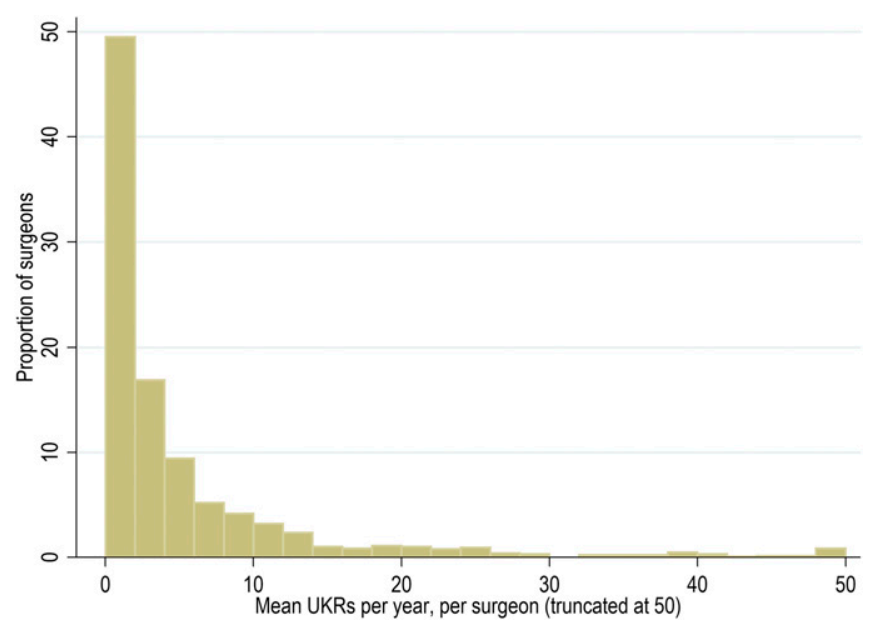

Fig. 1

Histogram demonstrating the distribution of UKR caseload among surgeons performing UKR in England and Wales. 
The Journal of Bone \& Joint Surgery $\cdot$ JBjS. org Volume 98-A • Number 1 · January 6, 2016

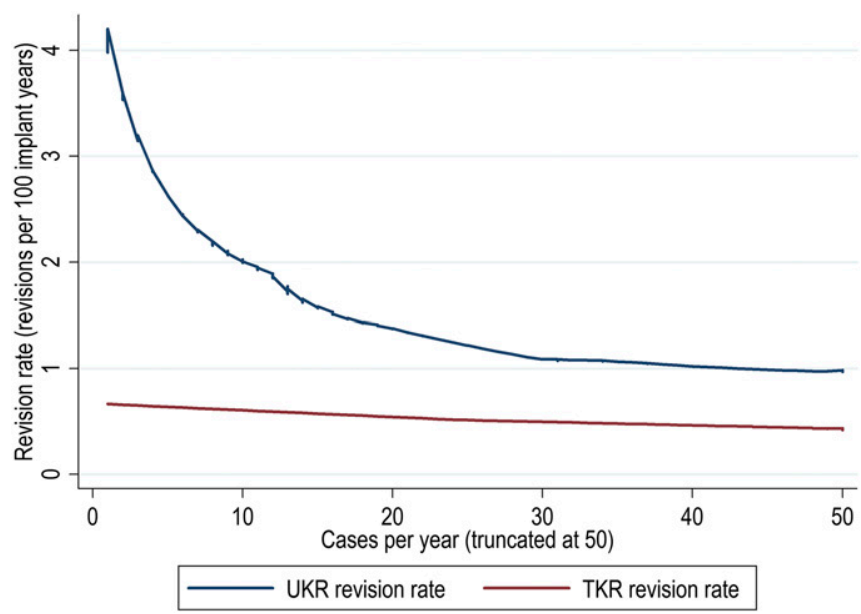

Fig. 2

LOWESS curve demonstrating the effect of increasing caseload on revision rates following UKR and TKR (up to fifty cases).

revision rate before the curve became shallower (at around ten cases per year); the revision rate plateaued at around thirty cases. For surgeons who performed fewer than ten cases per

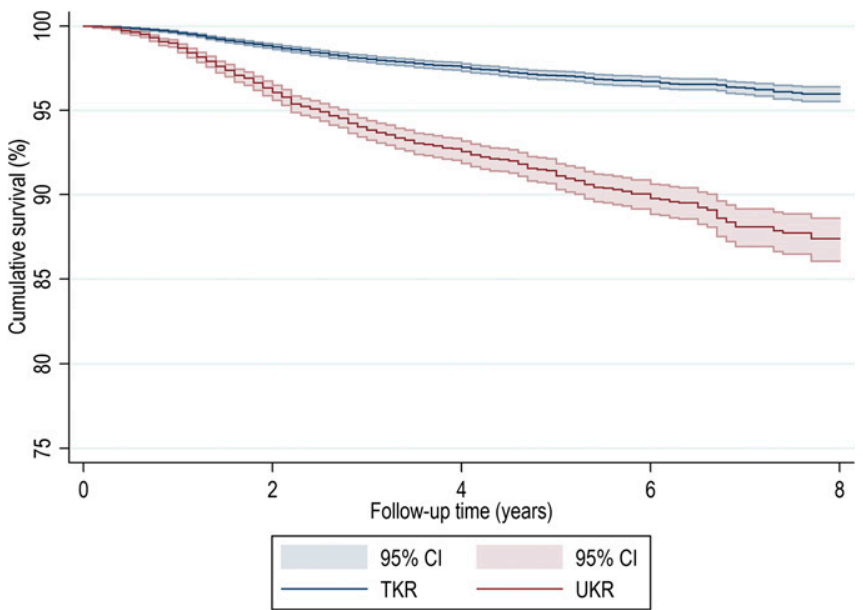

Fig. 3-A

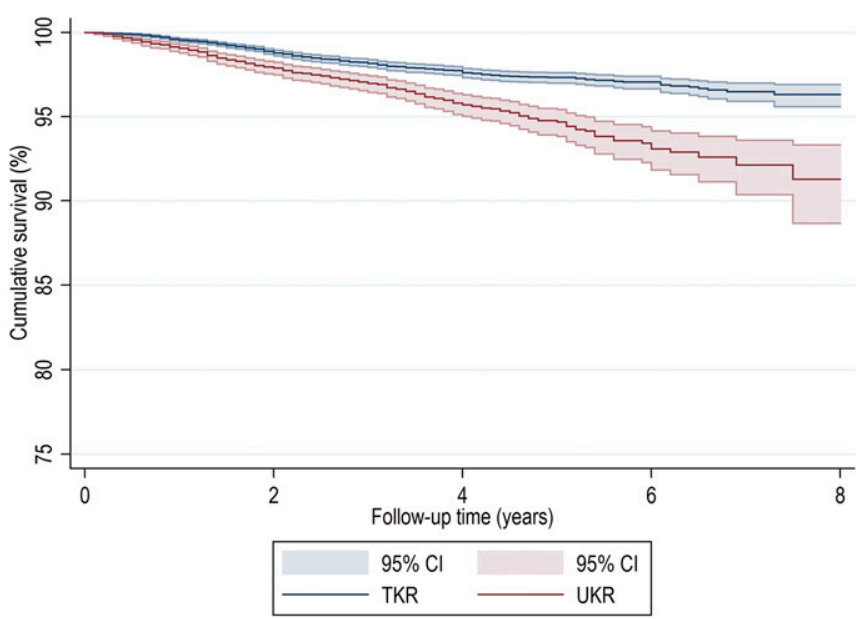

Effect of Surgical Caseload on Revision Rate After Total and Unicompartmental KneE Replacement

year, the mean revision rate was 2.52 per 100 implant-years (an eight-year survival rate of $87.9 \%$ [ $95 \% \mathrm{CI}=86.9 \%$ to $88.8 \%]$ ); this fell to 1.40 for surgeons who performed between ten and fewer than thirty cases per year $(90.1 \%[95 \% \mathrm{CI}=89.0 \%$ to $91.2 \%]$ ). With thirty cases or more per year, the revision rate was $0.98(92.4 \%[95 \% \mathrm{CI}=90.9 \%$ to $93.6 \%])$.

Linear splines were fitted, with knots placed at ten and thirty cases per year. The hazard ratio [HR] for revision was 0.86 ( $95 \% \mathrm{CI}=0.78$ to 0.94$)$ per five-case increase in annual caseload up to ten cases per year, $0.90(95 \% \mathrm{CI}=0.86$ to 0.93$)$ between ten and thirty cases, and $1.00(95 \% \mathrm{CI}=0.98$ to 1.02$)$ above thirty cases (Table II). With TKR, the effect of volume was effectively linear (Fig. 2). The HR for revision following TKR was 0.99 (95\% $\mathrm{CI}=0.98$ to 0.99$)$ for every five-case increase in annual caseload.

\section{Patient Characteristics}

Patient factors varied among the volume groups. Higher-volume surgeons operated on older patients, with more comorbidities but a lower level of deprivation (as measured with the Index of Multiple Deprivation [IMD], which is a U.K. governmental measure that assesses the level of deprivation in multiple domains [such as employment, income, and access to education] in

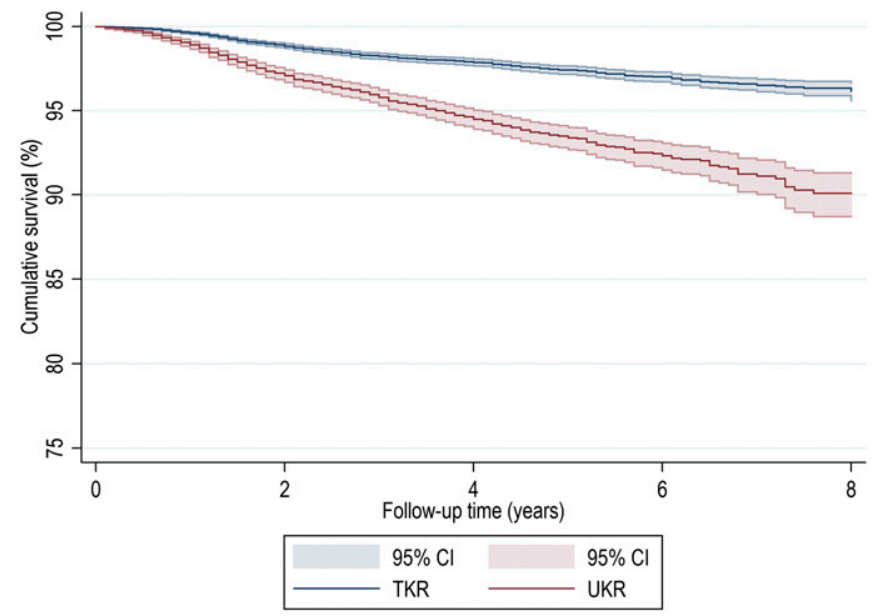

Fig. 3-B

Figs. 3-A, 3-B, and 3-C Matched survival comparisons, with revision (as defined by the National Joint Registry) as the end point, between the TKR group and the UKR low (Fig. 3-A), medium (Fig. 3-B), and high (Fig. 3-C)-volume groups. Additional details are given in Table IV. 
The Journal of Bone \& Joint Surgery • JBjS. org Volume 98-A • Number 1 · January 6, 2016

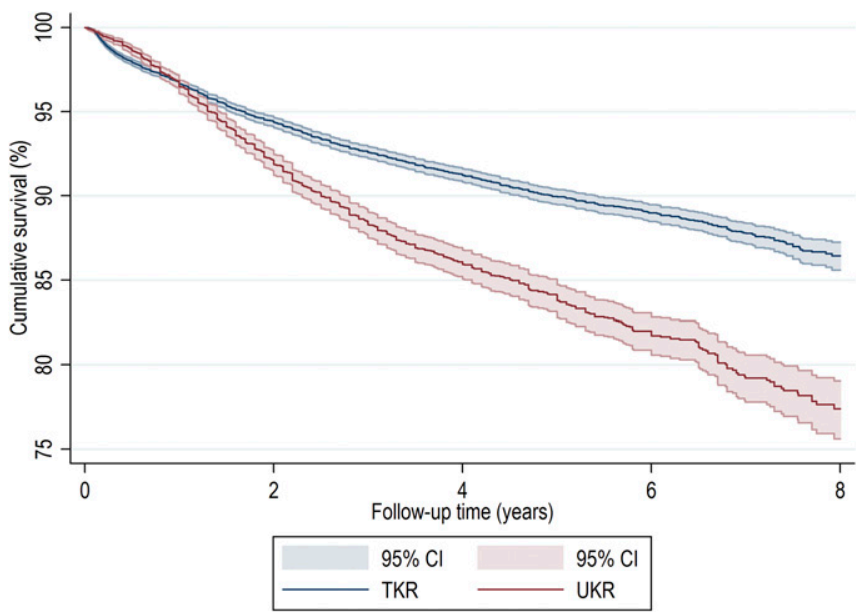

Fig. 3-D

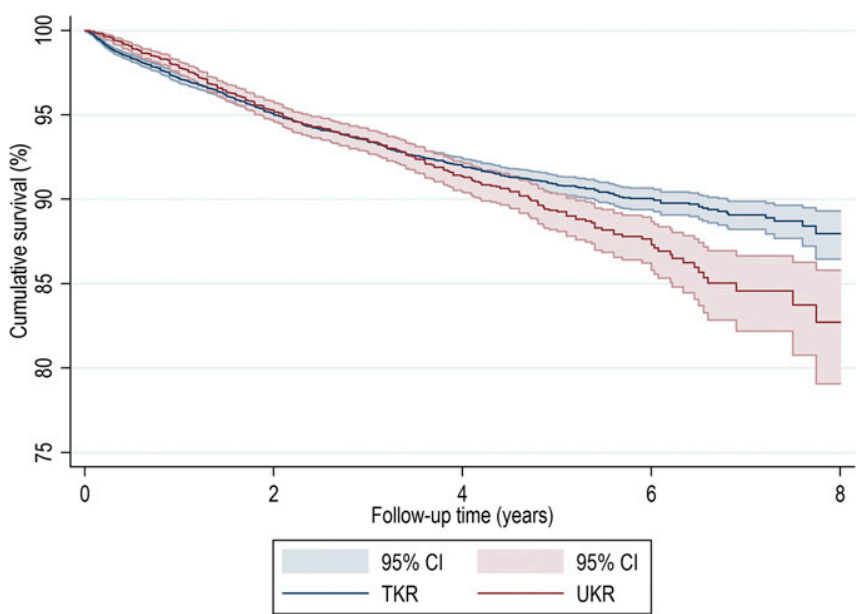

Fig. 3-F

small geographical areas ${ }^{29}$. While these differences were significant, the magnitude of the differences among the patients operated on in the different case-volume groups was small. Details of the patient characteristics in each group are given in Table I.

Reasons for revision differed among the groups. Surgeons with lower annual caseloads of UKRs were more likely to revise for aseptic loosening, unexplained pain, or malalignment (Table III).

\section{Comparison of Matched Patients}

UKRs were matched to TKRs at a 1:3 ratio. More than $95 \%$ of the UKRs were matched. Marked differences in the HR for revision and reoperation were observed between groups. For revision (as defined by the National Joint Registry), the HR was reduced from $3.19(95 \% \mathrm{CI}=2.84$ to 3.58$)$ in the low-volume group to $2.58(95 \% \mathrm{CI}=2.28$ to 2.93$)$ in the medium-volume group and $1.96(95 \% \mathrm{CI}=1.66$ to 2.32$)$ in the high-volume group. When both revisions and reoperations from the HES database were included, the HRs were reduced to $1.61(95 \% \mathrm{CI}=1.50$ to 1.73$)$ in the low-volume group and $1.32(95 \% \mathrm{CI}=1.22$ to 1.43) in the medium-volume group. The revision/reoperation rate following UKR in the high-volume group was similar to that after TKR ( $\mathrm{HR}=1.10,95 \% \mathrm{CI}=0.99$ to 1.22$)$.
Effect of Surgical Caseload on Revision Rate After

Total and Unicompartmental Knee Replacement

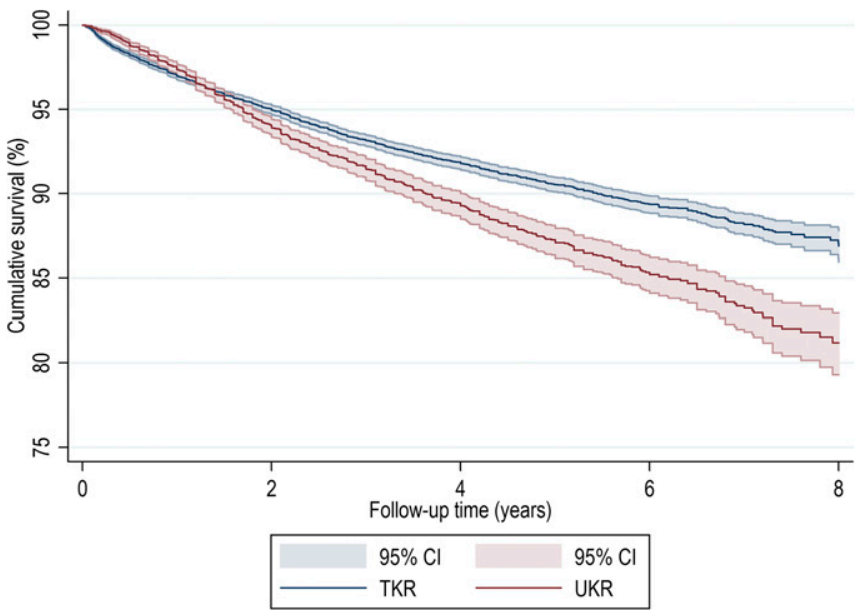

Fig. 3-E

Fig. 3-D, 3-E, and 3-F Matched survival comparisons, with revision/ reoperation (as determined with the HES data) as the end point, between the TKR group and the UKR low (Fig. 3-D), medium (Fig. 3-E), and high (Fig. 3-F)-volume groups. Additional details are given in Table IV.

Additional details are given in Table IV and Figures 3-A through 3-F.

\section{Discussion}

This study demonstrated an important effect of caseload on

1 the revision rate following UKR, with a fourfold difference in revision rate between the lowest and highest-caseload surgeons. While there was a caseload effect in TKR, it was not as marked as it was in UKR. Part of this effect is likely to be due to patient selection: low-volume surgeons tended to operate on younger, healthier patients who may have had earlier-stage disease. The observed differences in patient characteristics were small and are unlikely to account for the whole effect observed. However, the fact that higher-volume surgeons offer UKR to a greater proportion of their patients seeking knee replacement (implying broader patient-selection criteria) suggests that there may be differences in patient selection aside from those that were measurable with use of the data available in the databases that we examined. Low-volume surgeons revise UKRs for different reasons than high-volume surgeons, with low-volume surgeons being more likely to revise for loosening, unexplained pain, or malalignment. This could be interpreted as indicating 
The Journal of Bone \& Joint Surgery - Jbjs.org VOlume 98-A • Number 1 J January 6, 2016
Effect of Surgical Caseload on Revision Rate After Total and Unicompartmental Knee Replacement a higher rate of technical errors (leading to the higher rate of revision for malalignment and loosening) or that low-volume surgeons are more likely to revise a UKR in the absence of a clear, correctable cause of symptoms (as evidenced by the higher rate of revision for unexplained pain ${ }^{30}$. Patients who underwent a UKR by a high-volume surgeon (who performed thirty or more cases per year) had medium-term survival rates (in terms of revision/reoperation) that were similar to those for matched patients who underwent TKR.

To facilitate the comparison of the low, medium, and high-caseload groups, we used cut-points selected on the basis of the data. Other approaches would be to designate a certain proportion of surgeons as "high-volume" as was done by Robertsson et al. ${ }^{21}$ (who so designated the top quarter of units) or introduce clinically plausible cut-points a priori, the approach taken by the New Zealand Joint Registry ${ }^{31}$ (surgeons performing more than one case per month being considered "high volume") and by Baker et al. ${ }^{20}$ (who considered 100 cases or more over seven years to represent a high volume). In fact, each method resulted in similar thresholds: our low-volume threshold was ten cases per year compared with twelve cases per year ${ }^{6}$ or around fourteen cases per year (assuming participation in all years of the registry $)^{20}$.

However, the caseload effect is continuous. Revision rates do not plateau until the caseload has reached thirty cases per year (restricting the analysis to a small number of surgeons). Attempts to produce thresholds on the basis of continuous predictors are difficult and of questionable value ${ }^{32}$. As such, our thresholds should not be taken to represent either minimum or optimum figures for UKR caseload. While all surgeons performing fewer than thirty UKRs per year may improve their implant survival rates by increasing their caseload, the smoothed scatterplots (Fig. 2) suggest that very-low-volume users (those performing fewer than five cases per year) are of the greatest concern as they attain the worst results. This group (comprising more than half of all UKR users) may need to modify their practice by adopting the practices, in terms of patient selection and surgical technique, of larger units that report good results ${ }^{33}$. Better results can be obtained if, rather than many surgeons performing very few cases, fewer surgeons operate on larger numbers of patients each. Surgeons who wish to increase their caseload can do so by offering UKR to a greater proportion of their patients ${ }^{34}$ or by becoming a referral practice. Surgeons who are unable to achieve a sufficient caseload should abandon UKR and refer suitable patients elsewhere.
While the observed differences between the results of high and low-volume surgeons are partly explained by the surgical and patient factors explored in this study, there remains a substantial residual difference in implant survival among the caseload groups not explained by these factors. Other factors are likely to include more subtle patient factors (such as stage of disease) and surgical technique. UKR is more straightforward to revise than TKR, and it is likely that surgeons' thresholds for revising a poorly functioning UKR are lower than those for revising an equivalently poorly functioning $\mathrm{TKR}^{24}$. It is not clear, on the basis of this data set, whether the effect on implant survival observed in the medium term (at eight years) is maintained over the long term.

Future studies of outcomes of UKR should include the effect of caseload on patient-reported outcomes together with more detailed study of patient selection and technique. The study of preoperative and postoperative radiographs is likely to be important in determining the effect of these variables. Longerterm studies will provide greater clarity as to how long the caseload effect endures.

In conclusion, this study has confirmed the importance of surgical caseload in determining the survival of UKRs and, to a lesser extent, TKRs. The reasons for this effect are complex and not fully explained by variables recorded in the National Joint Registry; however, the patient selection and revision threshold of lower-volume surgeons may be factors. Examination of matched patients in this study demonstrated that surgeons performing a high volume of UKRs can achieve revision/reoperation rates similar to those observed following TKR.

\section{Appendix}

A A detailed description of the propensity score matching is eA available with the online version of this article as a data supplement at jbjs.org.

Alexander D. Liddle, DPhil, MRCS

Hemant Pandit, DPhil, FRCS(Orth)

Andrew Judge, $\mathrm{PhD}$

David W. Murray, MD, FRCS

Nuffield Department of Orthopaedics,

Rheumatology, and Musculoskeletal Sciences,

University of Oxford,

Windmill Road, Headington,

Oxford OX3 7LD, England.

E-mail address for D.W. Murray: david.murray@ndorms.ox.ac.uk

\section{References}

1. Mallory TH, Danyi J. Unicompartmental total knee arthroplasty. A five- to nine-year follow-up study of 42 procedures. Clin Orthop Relat Res. 1983 May;175:135-8.

2. National Joint Registry for England and Wales. 7th annual report 2010. 2010. http://www.njrcentre.org.uk/njrcentre/portals/0/njr\%207th\%20annual\%20report \%202010.pdf. Accessed 2015 Jul 29.

3. Brown NM, Sheth NP, Davis K, Berend ME, Lombardi AV, Berend KR, Della Valle CJ. Total knee arthroplasty has higher postoperative morbidity than unicompartmental knee arthroplasty: a multicenter analysis. J Arthroplasty. 2012 Sep;27(8)(Suppl): 86-90. Epub 2012 May 4.

4. Liddle AD, Judge A, Pandit $\mathrm{H}$, Murray DW. Adverse outcomes after total and unicompartmental knee replacement in 101,330 matched patients: a study of data from the National Joint Registry for England and Wales. Lancet. 2014 Oct 18;384 (9952):1437-45.

5. Lombardi AV Jr, Berend KR, Walter CA, Aziz-Jacobo J, Cheney NA. Is recovery faster for mobile-bearing unicompartmental than total knee arthroplasty? Clin Orthop Relat Res. 2009 Jun;467(6):1450-7. Epub 2009 Feb 19.

6. Liddle AD, Pandit H, Judge A, Murray DW. Patient-reported outcomes after total and unicompartmental knee arthroplasty: a study of 14,076 matched patients from the National Joint Registry for England and Wales. Bone Joint J. 2015 Jun;97-B(6):793-801. 7. Newman J, Pydisetty RV, Ackroyd C. Unicompartmental or total knee replacement: the 15-year results of a prospective randomised controlled trial. J Bone Joint Surg Br. 2009 Jan;91(1):52-7. 
The Journal of Bone \& Joint Surgery $\cdot$ JBjS. org VOlume 98-A • NUmber 1 · JanUary 6, 2016
Effect of Surgical Caseload on Revision Rate After

Total and Unicompartmental Knee Replacement
8. Australian Orthopaedic Association. National Joint Replacement Registry annual report 2014. 2014. https://aoanjrr.dmac.adelaide.edu.au/documents/10180/ 172286/Annual\%20Report\%202014. Accessed 2015 Jul 29.

9. National Joint Registry for England and Wales. 10th annual report 2013. 2013. http://www.njrcentre.org.uk/njrcentre/Portals/0/Documents/England/Reports/ 10th_annual_report/NJR\%2010th\%20Annual\%20Report\%202013\%20B.pdf. Accessed 2015 Jul 29

10. Danish Knee Arthroplasty Register. Annual report 2010. 2010. https:// www.knee.dk/groups/dkr/pdf/DKRreportEnglish2010.pdf. Accessed 2015 Jul 29.

11. The Swedish Knee Arthroplasty Register. Annual report 2012. 2012. http://www.myknee.se/pdf/117_SKAR_2012_Engl_1.0.pdf. Accessed 2015 Jul 29.

12. Foran JR, Brown NM, Della Valle CJ, Berger RA, Galante JO. Long-term survivorship and failure modes of unicompartmental knee arthroplasty. Clin Orthop Relat Res. 2013 Jan;471(1):102-8.

13. Heyse TJ, Khefacha A, Peersman G, Cartier P. Survivorship of UKA in the middleaged. Knee. 2012 Oct;19(5):585-91. Epub 2011 Oct 1.

14. Price AJ, Svard U. A second decade lifetable survival analysis of the Oxford unicompartmental knee arthroplasty. Clin Orthop Relat Res. 2011 Jan;469(1):

174-9.

15. Pandit H, Jenkins C, Gill HS, Barker K, Dodd CA, Murray DW. Minimally invasive Oxford phase 3 unicompartmental knee replacement: results of 1000 cases. J Bone Joint Surg Br. 2011 Feb;93(2):198-204.

16. Argenson JN, Blanc G, Aubaniac JM, Parratte S. Modern unicompartmental knee arthroplasty with cement: a concise follow-up, at a mean of twenty years, of a previous report. J Bone Joint Surg Am. 2013 May 15;95(10):905-9.

17. Edmondson MC, Isaac D, Wijeratna M, Brink S, Gibb P, Skinner P. Oxford unicompartmental knee arthroplasty: medial pain and functional outcome in the medium term. J Orthop Surg Res. 2011;6:52. Epub 2011 Oct 10.

18. Mercier N, Wimsey S, Saragaglia D. Long-term clinical results of the Oxford medial unicompartmental knee arthroplasty. Int Orthop. 2010 Dec;34(8):1137-43. Epub 2009 Oct 17.

19. Hamilton WG, Ammeen D, Engh CA Jr, Engh GA. Learning curve with minimally invasive unicompartmental knee arthroplasty. J Arthroplasty. 2010 Aug;25(5): 735-40. Epub 2009 Jul 4.

20. Baker P, Jameson S, Critchley R, Reed M, Gregg P, Deehan D. Center and surgeon volume influence the revision rate following unicondylar knee replacement: an analysis of 23,400 medial cemented unicondylar knee replacements. J Bone Joint Surg Am. 2013 Apr 17;95(8):702-9.
21. Robertsson $O$, Knutson $K$, Lewold $S$, Lidgren $L$. The routine of surgical management reduces failure after unicompartmental knee arthroplasty. J Bone Joint Surg Br. 2001 Jan;83(1):45-9.

22. Keys GW, UI-Abiddin Z, Toh EM. Analysis of first forty Oxford medial unicompartmental knee replacement from a small district hospital in UK. Knee. 2004 Oct;11(5):375-7.

23. Schroer WC, Barnes CL, Diesfeld P, LeMarr A, Ingrassia R, Morton DJ, Reedy M. The Oxford unicompartmental knee fails at a high rate in a high-volume knee practice. Clin Orthop Relat Res. 2013 Nov;471(11):3533-9. Epub 2013 Aug 2.

24. Goodfellow JW, O'Connor JJ, Murray DW. A critique of revision rate as an outcome measure: re-interpretation of knee joint registry data. J Bone Joint Surg Br. 2010 Dec;92(12):1628-31.

25. Lau RL, Perruccio AV, Gandhi R, Mahomed NN. The role of surgeon volume on patient outcome in total knee arthroplasty: a systematic review of the literature. BMC Musculoskelet Disord. 2012;13:250. Epub 2012 Dec 14.

26. Critchley RJ, Baker PN, Deehan DJ. Does surgical volume affect outcome after primary and revision knee arthroplasty? A systematic review of the literature. Knee. 2012 Oct;19(5):513-8. Epub 2012 Jun 5.

27. Singh JA, Kwoh CK, Boudreau RM, Lee GC, Ibrahim SA. Hospital volume and surgical outcomes after elective hip/knee arthroplasty: a risk-adjusted analysis of a large regional database. Arthritis Rheum. 2011 Aug;63(8):2531-9.

28. Cleveland WS. Robust locally weighted regression and smoothing scatterplots. J Am Stat Assoc. 1979 Dec;74(368):829-36.

29. Noble M, Wright G, Dibben C, Smith GAN, McLennan D, Anttila C, Barnes H, Mokhtar C, Noble S. The English indices of deprivation 2004. London: Queen's Printer and Controller of Her Majesty's Stationery Office; 2003.

30. Baker PN, Petheram T, Avery PJ, Gregg PJ, Deehan DJ. Revision for unexplained pain following unicompartmental and total knee replacement. J Bone Joint Surg Am. 2012 Sep 5;94(17):e126.

31. The New England Joint Registry. Fifteen year report. 2014 Oct. http://www. nzoa.org.nz/system/files/NZJR2014Report.pdf. Accessed 2015 Aug 26.

32. Altman DG, Lausen $B$, Sauerbrei W, Schumacher M. Dangers of using "optimal" cutpoints in the evaluation of prognostic factors. J Natl Cancer Inst. 1994 Jun 1;86 (11):829-35.

33. Liddle AD, Judge A, Pandit H, Murray DW. Determinants of revision and functional outcome following unicompartmental knee replacement. Osteoarthritis Cartilage. 2014 Sep;22(9):1241-50. Epub 2014 Jul 17.

34. Liddle AD, Pandit $H$, Judge A, Murray DW. Optimal usage of unicompartmental knee arthroplasty: a study of 41,986 cases from the National Joint Registry for England and Wales. Bone Joint J. 2015 Nov;97-B(11):1506-11. 